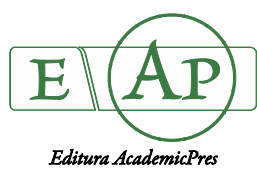

\title{
In Vitro Screening of Natural Drug Potentials for Mass Production
}

\author{
Gülsüm YALDIZ ${ }^{1 *}$, Ufuk KOCA ÇALIŞKAN ${ }^{2}$, Ceylan AKA² \\ ${ }^{1}$ Abant Izzet Baysal University, Faculty of Agriculture and Natural Sciences, Department of Field Crops, 14280 Bolu, \\ Turkey; gulsumyaldiz@ibu.edu.tr (*correspondingauthor) \\ ${ }^{2}$ Gazi University, Faculty of Pharmacy, Department of Pharmacognosy, 3 Tac Street, 06330 Ankara, \\ Turkey;ukoca@gazi.edu.tr; ceylanaka@gazi.edu.tr
}

\begin{abstract}
Methanolic extracts of 16 plant species, used in herbal and traditional medicinal preparations, collected from the same geographic region (Golcuk-Seben/Bolu, Turkey) were evaluated for their relative total phenolic contents, total antioxidant activities and their mineral contents in both dried plant samples and methanolic extracts. These species included Hypericum perforatum L., Thymus sipyleus Boiss., Equisetum arvense L., Achillea millefolium L., Tanacetum parthenium L., Thymus leucostomus Hausskn. \& Velen., Usnea longissima, Herniaria incana Boiss., Teucrium polium L., Stachys bithynica Boiss., Anthemis nobilis L., Sambucus ebulus L., Berberis vulgaris L., Malus communis Desf., Matricaria chamomilla L. and Scorzonera laciniata L. Spectrophotometric Folin-Ciocalteu and DPPH free radical scavenging activity methods were performed to detect the total phenolic contents and total antioxidant activities of the methanolic extracts, respectively. The results show that the fruits of Berberis vulgaris L., have the highest antioxidant activity and aerial parts of Tanacetum parthenium L. have the highest total phenolics (92.62\%). Mineral contents were determined by inductively coupled - mass spectrometry (ICP-MS). All dried plant samples and methanolic extracts contained high amounts of $\mathrm{Si}, \mathrm{Cu}, \mathrm{Fe}$ and $\mathrm{Ca}$. The highest levels of $\mathrm{Si}, \mathrm{Cu}, \mathrm{Fe}$ and $\mathrm{Ca}$ were found in Equisetum arvense L., Anthemis nobilis L., and Thymus sipyleus Boiss. dried plant samples, whereas Se was only detected in Usnea longissima. The highest levels of $\mathrm{Si}, \mathrm{Cu}, \mathrm{Fe}$ and $\mathrm{Ca}$ were found in the methanolic extracts of Usnea longissima, Anthemis nobilis L. and Thymus sipyleus Boiss. respectively.
\end{abstract}

Keywords: antioxidant, medicinal aromatic plants, mineral contents, phenolics

\section{Introduction}

Medicinal plants have a growing economic value in clinical, pharmaceutical, cosmeceutical, and other wide areas of international trade, although this varies widely among countries (Schmidt, 2012).

The plant extracts and phytochemicals isolated from these medicinal plants have been shown to exert biological activity in vitro and in vivo, which justified further research on traditional medicine focused on the characterization of their mostly antimicrobial and antioxidant activities (Deliorman Orhan $e t$ al., 2012). It is well accepted that, reactive oxygen compounds such as singlet oxygen, superoxide radicals, hydrogen peroxide, hydroxyl radicals and nitric oxide are unstable and extremely reactive (Ames et al.,1993). Oxidative stress-induced reactive oxygen species are considered to be the indicators of the development and progress of various diseases. Antioxidants prevent the negative impacts of free radicals and reactive oxygen species and protect the body (Gavamukulya et al., 2014). The widely known synthetic antioxidants are butylated hydroxyltoluene (BHT), butylated hydroxylanisole (BHA), propyl gallate and tertiary butylhydroquinone. However, the reliability of these synthetic antioxidants has been debated due to their toxic and carcinogenic effects, which can result in liver injury. Therefore, discovery of new and reliable antioxidants from natural resources has become a prominent research topic (Birman, 2012).

Flora of Gölcük-Seben, $100 \mathrm{~km}$ distance from Istanbul and Black sea region, which has been a little studied by scientific means before. This study provides an identification of potential, bioactive species that can be used as raw materials for plant derived products in several industries. The objectives of this study were to determine the relative total phenolic contents and antioxidant activity of 16 medicinal plant species including $H$. perforatum L., T. sipyleus Boiss., E. arvense L., $A$. millefolium L., T. parthenium L, T. leucostomus Hausskn. \& Velen., $U$. longissima, $H$. incana Boiss., $T$. polium L., $S$. bithynica Boiss., $A$. nobilis L., S. ebulus L., B. vulgaris L., $M$. communis Desf., $M$. chamomilla $\mathrm{L}$. and $S$. laciniata L. found in the Gölcük-Seben/Bolu area. The healing properties of these plants are very well known and used by local people in treatments of various diseases. Furthermore, some minerals, 
known to be useful for human health at certain dose levels, and which have a number of physiological functions in living organisms, were quantitatively determined both in dried plants and methanolic extracts. All these data will be used to help select the plant species, to be cultured in that region, if commercialization is warranted.

\section{Materials and Methods}

\section{Sitecharacterization}

Collection site is located in an area of Euro-Siberian, Mediterranean and Irano-Turanian Floristic Regions together with Sub-Euxine and Xero-Euxine zones. The coordination of research area is given in Table 2. The soil type of the research area is mainly brown forest soil, which is extremely calcified. The structure of the soil is fine-pored or granulated and the $\mathrm{pH}$ of the soil is alkaline and rarely neutral. The study site, dating back to upper-Eocene (35-40 mya) of the Tertiary in the Cenozoic era, consists of different types of rock, including basalt, andesite, turf and aglomera of a variety of colours from pink to black to claret red. The surface morphology, formed by erosion and tectonic activities, is elaborated by metamorphite and volcanic rocky types (Ikinci and Guneri, 2007). According to the data obtained from the Bolu Meteorology Station, the mean annual temperature of the area is expected to be between $7.1^{\circ} \mathrm{C}$ and $9.6^{\circ} \mathrm{C}$. Mean annual precipitation of the research area is expected to be between 599.6 and $869.6 \mathrm{~mm}$. Of this precipitation $32 \%$ is in winter, $29 \%$ is in spring, $21 \%$ is in autumn and $18 \%$ is in summer. The rainiest months of the year are December, January and February whereas the least rainy month is August.

\section{Plant materials and extraction of the plants}

A list of the studied plants, including the botanical name, voucher specimen and data related to therapeutic usage and the plant parts used are listed in Table 1. Voucher specimens were identified by Dr. Mecit Vural and they were deposited in the Herbarium of Field Crop Department, Abant İzzet Baysal University (Bolu, Turkey). Plant samples were air dried at room temperature and $20 \mathrm{~g}$ powdered plant materials extracted by chloroform and evaporated till dryness. Then $80 \%$ methanol $(2 \times 250 \mathrm{~mL})$ was added to the remaining material after chloroform filtration and again vaporized. Chloroform and methanolic extracts were used for further analyses.

\section{Determination of totalphenolic compounds}

Total phenolic contents of sixteen extracts were determined by using Folin-Ciocalteau Method (Singleton and Rossi, 1965). From each extract, $2 \mathrm{mg} \mathrm{mL}^{-1}$ sample was weighed and dissolved in 75\% ethanol. Reference gallic acid dilutions and each extract samples were mixed with $100 \mu \mathrm{L}$ Folin-Ciocalteau reagent, sodium carbonate $(20 \%)$ and distilled water. Mixtures were incubated for 30 minutes at $40^{\circ} \mathrm{C}$ and their absorbances were measured at $765 \mathrm{~nm}$. According to the calibration curve, the total phenolic concentration was calculated as gallic acid equivalents using the absorbance of the samples.

\section{Determination of total flavonoids}

To determine total flavonoids, $2 \mathrm{mg} \mathrm{mL}^{-1}$ of each extract samples and the reference quercetin solutions were mixed with $75 \% \mathrm{EtOH}, 10 \% \mathrm{AlCl}_{3}, 1 \mathrm{M}$ sodium acetate and $2,800 \mu \mathrm{L}$ distilled water separately. Mixtures were incubated for 30 minutes at room temperature and their absorbance was measured with a spectrophotometer at $415 \mathrm{~nm}$. According to calibration curves, total flavonoids were calculated as quercetin equivalents using the absorbance of the samples (Woisky and Salatino, 1998).

\section{Determination of radical scavenging activity}

The method of diphenyl picrylhydrazyl (DPPH) was utilized to determine in vitro radical scavenging activity (Burda and Oleszk, 2001). $1 \mathrm{mg} \mathrm{mL}^{-1}, 0.5 \mathrm{mg} \mathrm{mL}^{-1}, 0.25 \mathrm{mg} \mathrm{mL}^{-1}, 0.125$ $\mathrm{mg} \mathrm{mL} \mathrm{m}^{-1}, 0.0625 \mathrm{mg} \mathrm{mL}^{-1}$ of extracts and butylated hydroxyanisole reference (BHA) were prepared in methanol. $1 \mathrm{~mL}$ of each extract and BHA were mixed with $2 \mathrm{~mL}$ of DPPH ( $0.1 \mathrm{mM}$ in $70 \%$ methanol) and incubated for 30 minutes in the dark, at room temperature and their absorbances were measured at $517 \mathrm{~nm}$. Antiradical activity was calculated as follows: Scavenging activity $(\%)=(1-\mathrm{A} 1 / \mathrm{A} 0) \times 100$, where: A0: absorbance of control; A1: absorbance of sample.

\section{Determination of the concentration of minerals}

The mineral content in plant samples was determined using inductively coupled - mass spectrophotometry (Perkin Elmer Nexion 350 D / ICP-MS) according to the NMKL 186 method. Sample preparation was conducted by adding $8 \mathrm{~mL}$ $\mathrm{HNO}_{3}(65.0 \%)$ and $2 \mathrm{~mL}$ hydrogen peroxide (35.0\%) to a 0.25 g extract/plant sample and completing up to $50 \mathrm{~mL}$ by distilled pure water, in a microwave system (Berghof MWS4). The measurements were calculated as dry weight in $\mathrm{mg} / \mathrm{kg}$ according to the following formula:

$$
\mathrm{W}=[(\mathrm{a} \times \mathrm{V} \times \mathrm{F}) / \mathrm{m} \times 1000], \text { where: }
$$

$\mathrm{a}$ - is the content of the element in test solution in microgram per litre;

$\mathrm{V}$ - is the volume of the digestion solution after being made up, in milliliter;

$\mathrm{F}$ - is the dilution factor of the test solution;

$\mathrm{m}$ - is the initial sample mass, in gram.

\section{Results}

Antioxidant activities, total phenolic and flavonoid contents

In the present study, antioxidant activities were determined by using the DPPH method and the values varied between 99.53-19.65\% (Table 3). Berberis vulgaris fruits had the highest antioxidant activity (99.53\%) and it was followed by Achillea millefolium (97.21\%), Equisetum arvense (92.55\%) and Thymus leucostomus (91.63\%). Total phenolic contents varied between $92.62-1.35 \%$ the highest value in Tanacetum parthenium and the lowest value in Herniaria incana L. (Table 4). In the present study, Tanacetum parthenium (37.39\%) had the highest flavonoid contents and it was followed by Achillea millefolium (31.43\%), Matricaria chamomilla (21.03\%) and Thymussipyleus (20.56\%), respectively (Table 4).

\section{Mineralsubstance content}

The mean of the level of minerals determined in 16 medicinal plant samples is summarized in table 5-6. Se has important role on thyroid metabolism, improving and sustaining of nervous system (Georgieff, 2007; Skröder et al., 2015). Neurological problems have occurred due to deficiency 
Table 1. Chemical constituents of the 16 extracts with their traditional usage in Turkey

\begin{tabular}{|c|c|c|c|c|c|c|}
\hline $\begin{array}{l}\text { Scientific } \\
\text { name }\end{array}$ & Family & $\begin{array}{l}\text { Common } \\
\text { name }\end{array}$ & $\begin{array}{c}\text { Active } \\
\text { constituents }\end{array}$ & Therapeutic use & $\begin{array}{c}\text { Parts } \\
\text { analyzed }\end{array}$ & Reference \\
\hline Achillea millefolium L. & Asteraceae & Yarrow & $\begin{array}{l}\text { Phenolic acids, } \\
\text { tannins, } \\
\text { flavonoids, } \\
\text { essential oil, } \\
\text { sesquiterpene } \\
\text { lactones }\end{array}$ & $\begin{array}{c}\text { Stomachic, } \\
\text { spasmolytic, } \\
\text { diuretic, } \\
\text { anti-inflammatory }\end{array}$ & Aerial parts & Trouillasa et al., 2003 \\
\hline Anthemis nobilis $\mathrm{L}$. & Asteraceae & $\begin{array}{c}\text { Roman, } \\
\text { Chamomile } \\
\text { flower }\end{array}$ & $\begin{array}{l}\text { Volatile oils, } \\
\text { flavonoids, } \\
\text { coumarins, } \\
\text { phenolic acids }\end{array}$ & $\begin{array}{c}\text { Antispasmodic and } \\
\text { digestive; } \\
\text { food preservative }\end{array}$ & $\begin{array}{c}\text { Leaves and } \\
\text { Flowers }\end{array}$ & Gardiner, 1999 \\
\hline Berberis vulgaris $\mathrm{L}$. & Berberidaceae & Barberry & $\begin{array}{l}\text { Berberine, } \\
\text { berbamine, } \\
\text { palmitine, } \\
\text { jatrorrhizine, } \\
\text { isotetrandrine }\end{array}$ & $\begin{array}{c}\text { Sore throat, } \\
\text { fever, tanning skin, } \\
\text { antiviral }\end{array}$ & Fruit & Srivastava et al., 2015 \\
\hline Equisetum arvense $\mathrm{L}$. & Equisetaceae & Horsetail & $\begin{array}{l}\text { Phenolic acids, } \\
\text { flavonoids, sterols, } \\
\text { minerals, } \\
\text { alkaloids, } \\
\text { terpenoids, } \\
\text { saponins, } \\
\text { phytosterols, } \\
\text { aminoacids }\end{array}$ & $\begin{array}{c}\text { Diuretic, } \\
\text { antioxidant } \\
\text { vasorelaxant, } \\
\text { antinociceptive, } \\
\text { antiinflammatory }\end{array}$ & Aerial parts & Gallo et al., 2011 \\
\hline Herniaria incana Boiss. & Caryophyllaceae & $\begin{array}{l}\text { Rupture } \\
\text { worts }\end{array}$ & Terpenes & $\begin{array}{c}\text { Hypertension, kidney } \\
\text { antioxidant }\end{array}$ & Aerial parts & Parmer, 2012 \\
\hline $\begin{array}{c}\text { Hypericum } \\
\text { perforatum } \mathrm{L} .\end{array}$ & Hypericaceae & St John's wort & $\begin{array}{c}\text { Hyperforin, } \\
\text { adhyperforin, } \\
\text { hypericin, flavonol } \\
\text { glycosides }\end{array}$ & $\begin{array}{l}\text { Mild and moderate, } \\
\text { depression, epilepsy }\end{array}$ & Shoot & Briskin, 2000 \\
\hline Malus communis Desf. & Rosaceae & Apple & $\begin{array}{l}\text { Phenols, benzoic } \\
\text { acids, flavonoids, } \\
\text { phenyl propanoids }\end{array}$ & $\begin{array}{c}\text { Anti-microbial, } \\
\text { antiinflammator, } \\
\text { anti-mutagenic, anti- } \\
\text { carcinogenic, antiallergic, } \\
\text { anti-platelet, } \\
\text { vasodilatory actions }\end{array}$ & Fruit & Henríquez et al., 2010 \\
\hline $\begin{array}{c}\text { Matricaria chamomilla } \\
\text { L. }\end{array}$ & Asteraceae & $\begin{array}{l}\text { German } \\
\text { chamomile }\end{array}$ & $\begin{array}{l}\text { Essential oils, } \\
\text { sesquiterpene } \\
\text { lactones, pectic }\end{array}$ & $\begin{array}{c}\text { Antiinflammatory, } \\
\text { antispasmodic, } \\
\text { antioxidative, } \\
\text { antibacterial, antifungal, } \\
\text { anti-cancer, anti-allergic, } \\
\text { anti-pyretic }\end{array}$ & $\begin{array}{c}\text { Leaves and } \\
\text { flowers }\end{array}$ & $\begin{array}{l}\text { Heidari and Sarani } \\
2012\end{array}$ \\
\hline Sambucus ebulus L. & Caprifoliaceae & Dwarf Elder & $\begin{array}{l}\text { Dimeric and } \\
\text { mucin binding } \\
\text { lectins, protein } \\
\text { derivatives, } \\
\text { flavonoids, } \\
\text { linear Incision }\end{array}$ & $\begin{array}{l}\text { High fever, } \\
\text { rheumatic pains, } \\
\text { snake bites and wounds } \\
\text { treat burns, infectious } \\
\text { wounds, eczema, } \\
\text { urticaria, rheumatism, } \\
\text { inflammations }\end{array}$ & Fruits & $\begin{array}{l}\text { Shokrzadeh and } \\
\text { Saeedi Saravi } 2010\end{array}$ \\
\hline Scorzonera laciniata L. & Asteraceae & $\begin{array}{c}\text { Cutleaf } \\
\text { vipergrass }\end{array}$ & $\begin{array}{c}\text { Hexadecanoic } \\
\text { acid, } \\
\text { sesquiterpenoids }\end{array}$ & $\begin{array}{c}\text { Fever, carbuncle, } \\
\text { mastitis, arteriosclerosis, } \\
\text { kidney diseases, } \\
\text { hypertension, } \\
\text { diabetes mellitus } \\
\text { rheumatism }\end{array}$ & Aerial parts & Bosgelmez et al., 2007 \\
\hline $\begin{array}{c}\text { Stachys bithynica } \\
\text { Boiss. }\end{array}$ & Lamiaceae & Lamb's ear & $\begin{array}{l}\text { Tannins, phenolic } \\
\text { acids, flavonoids, } \\
\text { polyphenols }\end{array}$ & $\begin{array}{l}\text { Anti-inflammatory } \\
\text { genital tumor, } \\
\text { cancerous ulcer asthma, } \\
\text { moderate earaches pain, } \\
\text { cramps, dizziness, } \\
\text { fever, gout }\end{array}$ & Aerial parts & Vundac et al., 2007 \\
\hline $\begin{array}{c}\text { Tanacetum } \\
\text { parthenium L. }\end{array}$ & Asteraceae & Feverfew & $\begin{array}{c}\text { Sesquiterpene } \\
\text { lactones, } \\
\text { canin/artecanin }\end{array}$ & $\begin{array}{c}\text { Migraines, } \\
\text { inflammation, } \\
\text { arthritis }\end{array}$ & Aerial parts & Raal et al., 2014 \\
\hline
\end{tabular}




\begin{tabular}{|c|c|c|c|c|c|c|}
\hline Teucrium polium $\mathrm{L}$. & Lamiaceae & $\begin{array}{c}\text { Felty } \\
\text { Germander }\end{array}$ & $\begin{array}{l}\text { Tyrosol, } \\
\text { caffeic acid, } \\
\text { ferulic acid, } \\
\text { lutein } \\
\text { flavanoid }\end{array}$ & $\begin{array}{l}\text { Anti-inflammatory, anti- } \\
\text { nociceptive, anti- } \\
\text { bacterial, } \\
\text { anti-hypertensive, } \\
\text { hypolipidemic, anti- } \\
\text { heumatoid, } \\
\text { hypoglycemic } \\
\text { diuretic, diaphoretic, } \\
\text { tonic, antipyretic, } \\
\text { antispasmodic } \\
\text { cholagogic properties }\end{array}$ & $\begin{array}{l}\text { Leaves and } \\
\text { flowers }\end{array}$ & Sarac and Ugur, 2007 \\
\hline $\begin{array}{c}\text { Thymus } \\
\text { leucostomus } \\
\text { Hausskn. \& Velen. }\end{array}$ & Lamiaceae & Thyme & $\begin{array}{l}\text { Essential oil, } \\
\text { terpinene phenol }\end{array}$ & $\begin{array}{c}\text { Haemorrhoid, } \\
\text { rheumatism, stomach } \\
\text { kidney ailments. anti- } \\
\text { oxidative, antibacterial } \\
\text { antiseptic }\end{array}$ & Aerial parts & Yücel, 2011 \\
\hline Thymus sipyleus Boiss. & Lamiaceae & Thyme & $\begin{array}{l}\text { Essential oil, } \\
\text { terpinene phenol }\end{array}$ & $\begin{array}{l}\text { Haemorrhoid, } \\
\text { rheumatism, stomach } \\
\text { kidney ailments. anti- } \\
\text { oxidative, antibacterial, } \\
\text { antiseptic, antifungal }\end{array}$ & Aerial parts & $\begin{array}{c}\text { Yiğit and Kandemir, } \\
2002\end{array}$ \\
\hline Usnea longissima & Usneaceae & $\begin{array}{l}\text { Methuselah's } \\
\text { beard }\end{array}$ & $\begin{array}{c}\text { Phenolic } \\
\text { compounds, } \\
\text { longissiminone, } \\
\text { Glutinol }\end{array}$ & $\begin{array}{l}\text { Pain relief fever control, } \\
\text { lower respiratory } \\
\text { infections, anti- } \\
\text { inflammatory, } \\
\text { analgesic, antipyretic, } \\
\text { anti-tumor, anti } \\
\text { cholesterol, } \\
\text { nematocidal properties }\end{array}$ & Whole plant & Altınterim, 2012 \\
\hline
\end{tabular}

Table 2. Collection sources of the plant material

\begin{tabular}{clcc}
\hline $\begin{array}{c}\text { Sample } \\
\text { no. }\end{array}$ & \multicolumn{1}{c}{ Species } & Altitude $(\mathrm{m})$ & Coordinates \\
\hline 1 & Achillea millefolium $\mathrm{L}$. & $1400-1550$ & $40.497429,31.607366$ \\
2 & Anthemis nobilis $\mathrm{L}$ & 1100 & $40.674996,31.634132$ \\
3 & Berberis vulgaris $\mathrm{L}$. & $1170-1200$ & $40.659762,31.636621$ \\
4 & Equisetum arvense $\mathrm{L}$. & 900 & $40.669235,31.635162$ \\
5 & Herniaria incana Boiss. & 1400 & $40.655432,31.634690$ \\
6 & Hypericum perforatum $\mathrm{L}$. & $1100-1200$ & $40.674996,31.634132$ \\
7 & Malus communis Desf. & 1010 & $40.453554,31.591659$ \\
8 & Matricaria chamomilla $\mathrm{L}$. & 900 & $40.669235,31.635162$ \\
9 & Sambucus ebulus L. & 900 & $40.669235,31.635162$ \\
10 & Scorzonera laciniata $\mathrm{L}$. & $1100-1200$ & $40.674996,31.634132$ \\
11 & Stachys bithynica Boiss. & 1080 & $40.674996,31.634132$ \\
12 & Tanacetum parthenium $\mathrm{L}$. & 1080 & $40.674996,31.634132$ \\
13 & Teucrium polium L. & $1350-1450$ & $40.585104,31.633273$ \\
14 & Thymus leucostomus Hausskn. \& Velen. & 1110 & $40.462697,31.595006$ \\
15 & Thymus sipyleus Boiss. & 1110 & $40.462697,31.595006$ \\
16 & Usnea longissima & $1350-1450$ & $40.585104,31.633273$ \\
\hline
\end{tabular}

of it (Georgieff, 2007). Only U. longissima dried plant sample contained Se (220.11 $\pm 44.02 \mathrm{ppm})$ The WHO has advised iron's grade in plants as $20 \mathrm{mg} \mathrm{kg}^{-1}$ (Shah et al., 2011), which has positive effects on strengthening the immune system and cognitive performance (Domellöf et al., 2001; Kon et al., 2010). Low levels of iron gives rise to inflammation, pain, loss of appetite, dysphagia, exhaustion, cold limbs and impairment in nails, on the other hand, high iron intake causes lethargy, increasing blood flow of vessel and hypertension. The highest Fe content was found in Thymus sipyleus (3,369.20 \pm 438.00 $\mathrm{ppm}$ ) and it was respectively followed by Thymus leucostomus (1,246.02 $\pm 161.98 \mathrm{ppm})$, S. potentilla (912.57 $\pm 118.63 \mathrm{ppm})$, and Matricaria chamomilla (830.07 $\pm 107.9 \mathrm{ppm})$. Silicon has affected bone, keratinous structures, and immune system. The
Si content in dried plant samples changed from $1,176.09$ to $32.13 \pm 4.2 \mathrm{ppm}$; with the highest value in $E$. arvense and the lowest value in $M$. communis. Calcium $(\mathrm{Ca})$ is necessary for enzyme activity (Berridge, 1975), and has role on skeletal structure. Deficiency of Ca causes convulsion on muscles, and lumbago (Passwater, 1983), however, excess intake of Ca triggers gastrointestinal diseases such as dyspepsia, mouth dryness caused by hyperkalemia; weakens of physical activity (Watts, 1990). Total Ca concentrations in the dried plants ranged from $0.08 \pm 0.01$ to $4.74 \pm 0.66$, with the highest value in $E$. arvense and the lowest value in $M$. communis. Copper has an antioxidant activity (Tsuji et al., 2016) and its Daily recommended intake is $10 \mathrm{mg} \mathrm{kg}{ }^{-1}$ (Hassan et al., 2012). Excessive amount of $\mathrm{Cu}$ uptake gives rise to pain, irritation, 
296

Table 3. Free radical scavenging activity (DPPH)

\begin{tabular}{|c|c|c|c|c|}
\hline Sample & $1 \mathrm{mg} / \mathrm{mL}$ & $0.5 \mathrm{mg} / \mathrm{mL}$ & $0.25 \mathrm{mg} / \mathrm{mL}$ & $0.125 \mathrm{mg} / \mathrm{mL}$ \\
\hline BHA (control) & 98.14 & 97.67 & 96.28 & 94.88 \\
\hline Achillea millefolium $\mathrm{L}$. & 97.21 & 88.84 & 88.37 & 87.91 \\
\hline Anthemis nobilis $\mathrm{L}$. & 68.37 & 46.51 & 45.12 & 43.72 \\
\hline Berberis vulgaris $\mathrm{L}$. & 99.54 & 74.88 & 53.023 & 47.44 \\
\hline Equisetum arvense $\mathrm{L}$. & 92.56 & 89.30 & 82.323 & 64.19 \\
\hline Herniaria incana Boiss. & 67.91 & 63.26 & 56.28 & 38.14 \\
\hline Hypericum perforatum $\mathrm{L}$. & 77.67 & 88.37 & 94.88 & 95.81 \\
\hline Malus communis Desf. & 84.65 & 72.09 & 63.72 & 39.07 \\
\hline Matricaria chamomilla $\mathrm{L}$. & 85.58 & 74.88 & 59.07 & 58.14 \\
\hline Sambucus ebulus L. & 85.58 & 83.72 & 76.28 & 51.63 \\
\hline Scorzonera laciniata L. & 73.95 & 69.767 & 66.51 & 57.21 \\
\hline Stachys bithynica Boiss. & 80 & 74.883 & 61.86 & 60.93 \\
\hline Tanacetum parthenium $\mathrm{L}$. & 83.72 & 81.860 & 77.21 & 63.72 \\
\hline Teucrium polium $\mathrm{L}$. & 47.90 & 38.60 & 29.767 & 19.65 \\
\hline $\begin{array}{l}\text { Thymus leucostomus } \\
\text { Hausskn. \& Velen. }\end{array}$ & 91.63 & 66.51 & 65.581 & 30.23 \\
\hline Thymus sipyleus & 13.02 & 12.56 & - & - \\
\hline Usnea longissima & 51.16 & 44.19 & 42.33 & 26.98 \\
\hline
\end{tabular}

Table 4. Total flavonoid and phenolic contents

\begin{tabular}{|c|c|c|}
\hline Sample & Total flavonoid \% & Phenolic content $\%$ \\
\hline Achillea millefolium L. & $22.248 \pm 7.96$ & $18.878 \pm 1.5$ \\
\hline Anthemis nobilis $\mathrm{L}$. & $12.266 \pm 0.22$ & $13.366 \pm 0.88$ \\
\hline Berberis vulgaris $\mathrm{L}$. & $4.780 \pm 0.05$ & $14.552 \pm 0.87$ \\
\hline Equisetum arvense $\mathrm{L}$. & $5.363 \pm 0.009$ & $1.731 \pm 0.0$ \\
\hline Herniaria incana Boiss. & $6.043 \pm 0.22$ & $1.346 \pm 0.35$ \\
\hline Hypericum perforatum $\mathrm{L}$. & $8.567 \pm 0.16$ & $10.641 \pm 0.47$ \\
\hline Malus communis Desf. & $2.7476 \pm 0.13$ & $21.603 \pm 0.78$ \\
\hline Matricaria chamomilla $\mathrm{L}$. & $20.711 \pm 0.39$ & $8.782 \pm 0.43$ \\
\hline Sambucus ebulus L. & $6.084 \pm 0.2$ & $10.128 \pm 0.15$ \\
\hline Scorzonera laciniata $\mathrm{L}$. & $6.913 \pm 0.45$ & $7.982 \pm 0.19$ \\
\hline Stachys bithynica Boiss. & $8.941 \pm 0.17$ & $39.038 \pm 1.11$ \\
\hline Tanacetum parthenium $\mathrm{L}$. & $35.986 \pm 1,24$ & $92.628 \pm 5.44$ \\
\hline Teucrium polium $\mathrm{L}$. & $15.457 \pm 0.65$ & $21.731 \pm 1.15$ \\
\hline Thymus leucostomus Hausskn. \& Velen. & $13.523 \pm 0.47$ & $31.059 \pm 1.5$ \\
\hline Thymus sipyleus & $19.735 \pm 0.72$ & $45.737 \pm 1.52$ \\
\hline Usnea longissima & $2.975 \pm 0.07$ & $0.160 \pm 0.05$ \\
\hline
\end{tabular}

Table 5. Mineral content of dried plant samples

\begin{tabular}{|c|c|c|c|c|c|}
\hline Sample & Se (ppb) & Si (ppm) & $\mathrm{Cu}(\mathrm{ppm})$ & $\mathrm{Fe}(\mathrm{ppm})$ & $\mathrm{Ca}(\%)$ \\
\hline Achillea millefolium $\mathrm{L}$. & ND & $418.60 \pm 62.79$ & $9.43 \pm 1.23$ & $110.84 \pm 14.41$ & $1.13 \pm 0.16$ \\
\hline Anthemis nobilis $\mathrm{L}$. & ND & $438.67 \pm 65.80$ & $13.18 \pm 1.71$ & $442.52 \pm 57.53$ & $1.53 \pm 0.21$ \\
\hline Berberis vulgaris $\mathrm{L}$. & ND & $83.44 \pm 12.52$ & $10.55 \pm 1.37$ & $40.38 \pm 5.25$ & $0.20 \pm 0.03$ \\
\hline Equisetum arvense $\mathrm{L}$. & ND & $1,176.09 \pm 176.41$ & $3.21 \pm 0.42$ & $855.42 \pm 11.20$ & $4.74 \pm 0.66$ \\
\hline Herniaria incana Boiss. & ND & $1,163.27 \pm 2,950.67$ & $6.93 \pm 0.90$ & $571.62 \pm 74.31$ & $2.10 \pm 0.29$ \\
\hline Hypericum perforatum & ND & $268.58 \pm 40.29$ & $7.91 \pm 1.03$ & $169.53 \pm 22.04$ & $0.44 \pm 0.06$ \\
\hline Malus communis Desf. & ND & $32.13 \pm 4.82$ & $2.24 \pm 0.29$ & $15.78 \pm 2.05$ & $0.08 \pm 0.01$ \\
\hline Matricaria chamomilla & ND & $243.56 \pm 36.53$ & $10.93 \pm 1.42$ & $830.07 \pm 107.91$ & $1.77 \pm 0.25$ \\
\hline Sambucus ebulus L. & ND & $106.59 \pm 15.99$ & $1.84 \pm 0.24$ & $32.53 \pm 4.23$ & $0.44 \pm 0.06$ \\
\hline Scorzonera laciniata L. & ND & $382.64 \pm 57.40$ & $11.73 \pm 1.53$ & $912.57 \pm 118.63$ & $3.41 \pm 0.48$ \\
\hline Stachys bithynica Boiss. & ND & $604.72 \pm 90.71$ & $12.19 \pm 1.58$ & $590.57 \pm 76.77$ & $2.89 \pm 0.40$ \\
\hline Tanacetum parthenium & ND & $391.10 \pm 58.66$ & $13.06 \pm 1.70$ & $121.72 \pm 15.82$ & $1.24 \pm 0.17$ \\
\hline Teucrium polium $\mathrm{L}$. & ND & $689.35 \pm 103.40$ & $11.13 \pm 1.45$ & $680.57 \pm 88.7$ & $1.33 \pm 0.19$ \\
\hline Thymus leucostomus Hausskn. \& Velen. & ND & $751.35 \pm 112.70$ & $7.88 \pm 1.02$ & $1,246.02 \pm 161.98$ & $1.90 \pm 0.27$ \\
\hline Thymus sipyleus & ND & $888.9 \pm 133.26$ & $4.75 \pm 0.62$ & $3,369.20 \pm 438.00$ & $1.97 \pm 0.28$ \\
\hline Usnea longissima & $220.11 \pm 44.02$ & $1,048.08 \pm 157.21$ & $3.19 \pm 0.41$ & $410.74 \pm 53.40$ & $0.79 \pm 0.11$ \\
\hline
\end{tabular}

ND: Non-detectable. 
Table 6. Mineral content of the methanolic extracts

\begin{tabular}{|c|c|c|c|c|c|}
\hline Sample & $\mathrm{Se}(\mathrm{ppb})$ & $\mathrm{Si}(\mathrm{ppm})$ & $\mathrm{Cu}(\mathrm{ppm})$ & $\mathrm{Fe}(\mathrm{ppm})$ & $\mathrm{Ca}(\%)$ \\
\hline Achillea millefolium $\mathrm{L}$. & ND & $227.02 \pm 34.05$ & $16.27 \pm 2.12$ & $33.05 \pm 4.30$ & $0.10 \pm 0.01$ \\
\hline Anthemis nobilis L. & ND & $279.78 \pm 41.97$ & $51.57 \pm 6.70$ & $15.91 \pm 2.07$ & $0.07 \pm 0.01$ \\
\hline Berberis vulgaris L. & ND & $27.92 \pm 4.19$ & $5.60 \pm 0.73$ & $8.41 \pm 1.09$ & $0.02 \pm 0.03$ \\
\hline Equisetum arvense $\mathrm{L}$. & ND & $334.08 \pm 50.11$ & $10.15 \pm 1.32$ & $135.23 \pm 17.58$ & $0.54 \pm 0.08$ \\
\hline Herniaria incana Boiss. & ND & $270.02 \pm 40.50$ & $10.72 \pm 1.39$ & $79.99 \pm 10.40$ & $0.19 \pm 0.03$ \\
\hline Hypericum perforatum & ND & $155.84 \pm 23.38$ & $19.44 \pm 2.53$ & $34.85 \pm 4.53$ & $0.13 \pm 0.02$ \\
\hline Malus communis Desf. & ND & $121.38 \pm 18.21$ & $1.78 \pm 0.23$ & $6.45 \pm 0.84$ & $0.06 \pm 0.01$ \\
\hline Matricaria chamomilla. & ND & $152.94 \pm 22.94$ & $10.96 \pm 1.42$ & $25.29 \pm 3.29$ & $0.05 \pm 0.01$ \\
\hline Sambucus ebulus L. & ND & $67.61 \pm 10.14$ & $2.66 \pm 0.35$ & $6.97 \pm 0.91$ & $0.03 \pm 0.04$ \\
\hline Scorzonera laciniata L. & ND & $154.08 \pm 23.11$ & $9.88 \pm 1.28$ & $41.43 \pm 5.39$ & $0.26 \pm 0.04$ \\
\hline Stachys bithynica Boiss. & ND & $385.51 \pm 57.83$ & $16.17 \pm 2.10$ & $121.14 \pm 15.75$ & $0.35 \pm 0.05$ \\
\hline Tanacetum parthenium & ND & $126.75 \pm 19.01$ & $20.82 \pm 2.71$ & $15.77 \pm 2.05$ & $0.10 \pm 0.01$ \\
\hline Teucrium polium L. & ND & $335.48 \pm 50.32$ & $21.33 \pm 2.77$ & $60.66 \pm 7.89$ & $0.24 \pm 0.03$ \\
\hline Thymus leucostomus Hausskn. \& Velen. & $386.78 \pm 77.36$ & $358.13 \pm 53.72$ & $10.43 \pm 1.36$ & $70.91 \pm 9.22$ & $0.19 \pm 0.03$ \\
\hline Thymus sipyleus & ND & ND & $12.79 \pm 1.66$ & $596.35 \pm 77.53$ & $0.93 \pm 0.13$ \\
\hline Usnea longissima & $746.02 \pm 149.20$ & $568.54 \pm 85.28$ & $2.37 \pm 0.31$ & $18.71 \pm 24.33$ & $0.04 \pm 0.06$ \\
\hline
\end{tabular}

anemia, diarrhea and organ damage (brain, kidney, liver etc.), (Water Treatment Solutions (Cu), 1998; Adnan Iqbal et al., 2011; Mebrahtu and Zerabruk, 2011; Aenab et al., 2013) however, deficiency of copper causes arthrosis and cardiovasculer problems and, susceptibility to infection (Passwater, 1983; Klevay, 1975; Passwater, 1983; Campbell, 2001). A. nobilis, T. parthenium, S. bithynica and S. potentilla also contained significant amounts of $\mathrm{Cu}(1.84 \pm 0.24-13.06$ $\pm 1.70)$. In addition, $A$. nobilis $\mathrm{L}$. methanolic extract included more copper than dried plant sample.

In metanolic plant extract, $T$. leucostomus and $U$. longissima contained selenium $(386.78 \pm 77.36-746.02 \pm 149.20 \mathrm{ppm})$ respectively. Although, dried plant samples demonstrated lower levels of Se, methanolic extracts had higher levels of Se that might due to the water solubility of the Se mineral. The highest Fe content had T. sipyleus $(596.35 \pm 77.53 \mathrm{ppm})$ and it was respectively followed by $E$. arvense (135.23 $\pm 17.58 \mathrm{ppm})$, S. bithynica (121.14 $\pm 15.75 \mathrm{ppm})$, H. incana $(79.99 \pm 10.40$ $\mathrm{ppm})$. The amount of $\mathrm{Si}$ content in exctract plant samples changed from $568.54 \pm 85.28$ to $27.92 \pm 4.19 \mathrm{ppm}$; with the highest value in $U$. longissima and the lowest value in $B$. vulgaris. Total $\mathrm{Ca}$ concentrations in the extract of plants ranged from $0.023 \pm 0.003$ to $0.93 \pm 0.13$, with the highest value in $T$. sipyleus and the lowest value in B. vulgaris. In addition, $A$. nobilis, $T$. polium and $T$. parthenium also contained significant amounts of $\mathrm{Cu}(51.57 \pm 6.70-21.33 \pm$ $2.77-20.82 \pm 2.71$ ). Furthermore, dry plant samples contained higher mineral elements than methanolic plant extracts.

\section{Discussion}

In previous literatures, total flavonoid, phenolic content and antioxidant activity of $H$. incana and $T$. parthenium reported are in agreement with the result of the present study (Rabiei et al., 2014; Wu et al., 2006). Studies on A. millefolium demonstrated the presence of flavonoids such as apigenin, luteolin and rutin, which show powerful antioxidant properties, in this plant (Tuberoso et al., 2009; Kocevar et al., 2012). Hanachi and Sh (2009) reported that the antioxidant activity of the ethanolic extract of $B$. vulgaris fruit has the highest $(27.26 \pm 1.07 \%)$. Our data indicate that $T$. polium and $U$. longissima have moderate levels of antioxidant activity in agreement with the fact that polyphenols and chemical composition are affected by different factors, such as genotype, environmental conditions and extraction procedures (Halici $e t$ al., 2005; Sharififar et al., 2009; Stankovic et al., 2012; Andrè et al., 2012). Ahmed et al. (2016) reported that the species such as A. millefolium L., Equisetum arvense L, $H$. perforatum L., $S$. aromaticum L., M. chamomilla L. exhibited high antioxidant properties. Kukric et al. (2013) evaluated the antioxidative effect of dried ethanolic extract of $E$. arvense, which had an $\mathrm{IC}_{50}$ and AAI (antioxidant activity index) of 13.5 and 3.9, respectively. Although present findings are somehow similar to results of those earlier studies, differences in extraction and activity methods, climate, soil, environmental factors, diseases, and pesticide treatments, harvest time, drying and storage methods and plant parts used in analyses may significantly affect the antioxidant activity of plants (Bergonzi $e t$ al., 2001; Wang and Zheng, 2001). In previous scientific studies, all over the world on nutritive composition of wild plants high quantities of minerals, especially $\mathrm{K}, \mathrm{Na}, \mathrm{Ca}, \mathrm{P}, \mathrm{Mg}$ (Guil Guerrero et al., 1998; Agrahar-Murugkar and Subbulakshmi, 2005) are indicated. Iron values were reported between 10 and $981 \mathrm{mg} \mathrm{kg}^{-1}$ with highest value determined in sage (Zengin $e t$ al., 2008). Se values were reported between 0.0015 and $0.4 \mathrm{mg}$ $\mathrm{kg}^{-1}$ with the highest value determined in Solenostemma arghel (Sheded, 2006). Average values for copper content of some edible plants varied from $0.05-18.4 \mathrm{mg} \mathrm{kg}^{-1}$ (Yildirim et al., 2001; Turan et al., 2003). Equisetum arvense had minerals content such as silica, calcium, magnesium, selenium, iron, potassium, zinc (Veit et al., 1995; Beckert et al., 1997). Ozcan (2004) indicated that the highest levels of $\mathrm{Ca}, \mathrm{Fe}, \mathrm{K}, \mathrm{Mg}$ and S were found in T. vulgaris, L. officinalis $\mathrm{L}$., $A$. graveolens $\mathrm{L}$., $O$. basilicum $\mathrm{L}$ and $S$. alba $\mathrm{L}$, respectively while $\mathrm{Bi}, \mathrm{Cd}, \mathrm{Li}, \mathrm{Pb}$ and Se contents of condiments were found to be very low. Our studies on mineral content of the plants were also in accordance with the previous findings. 
298

\section{Conclusions}

In the present study, it was determined that some plants in Bolu/Turkey contained abundant phenolic compounds and mineral constituents. Antioxidant activities were also evaluated from methanolic extracts of these plants. From the experimental results, T. sipyleus and Stachys bithynica Boiss with $A$. nobilis L., E. arvense L., T. parthenium L, T. polium L., $T$. leucostomus Hausskn. \& Velen were of particular interest. If these plants are protected by culturing, then, mass production of natural drug potential can be carried out easily and efficiently.

\section{Acknowledgements}

This work was supported by Scientific Research Project Fund (2014.10.07.768), Faculty of Agriculture and Natural Sciences, Abant Izzet Baysal University, Turkey.

\section{References}

Agrahar-Murugkar A, Subbulakshmi G (2005). Nutritive values of wild edible fruits, berries, nuts, roots and species consumed by the Khasi tribes of India. Ecology Food and Nutrition 44:207-223.

Ahmed S, Hasan MM, Mahmood ZA (2016). Antiurolithiatic plants: Multidimensional Pharmacology. Journal of Pharmacognosy and Phytochemistry 5(2):04-24.

Akram, R, Hassan G, Seyed-Mostafa HZ, Fariba M, Abdolkarim C, Tayebeh S (2015). Antioxidant effects of Matricaria chamomilla $\mathrm{L}$. in paraquat induced kidney oxidative damage in rats. Pharmacologia $6: 45-51$.

Altınterim B (2012). Allergic and antiallergic effects of nettle leaf. Lokman Hekim Journal 2(1):47-49.

Ames BN, Shigenaga MK, Hagen TM (1993). Oxidants, antioxidants, and the degenerative diseases of aging. Proceedings of the National Academy of Sciences 90:7915-7922.

Andrè CM, Oufir M, Hoffmann L, Hausman JF, Rogez H, Larondelle Y, Evers D (2009). Influence of environment and genotype on polyphenol compounds and in vitro antioxidant capacity of native Andean potatoes (Solanum tuberosum L.). Journal of Food Composition and Analysis 22:517-524.

Baharfar R, Azimi R, Mohseni M (2015). Antioxidant and antibacterial activity of flavonoid-, polyphenol and anthocyanin-rich extracts from Thymus kotschyanus boiss \& hohen aerial parts. Journal of Food Science and Technology 52(10):6777-6783.

Beckert C, Horn C, Schnitzler JP, Lehning A, Heller W, Veit M (1997). Styrylpyrone biosynthesis in Equisetum arvense. Phytochemistry 44(2):275-283.

Bergonzi MC, Bilia AR, Gallori S, Guerrini D, Vincieri FF (2001). Variability in the content of the constituents of Hypericum perforatum L. and some commercial extracts. Drug Development and Industrial Pharmacy 27:491-497.

Berridge M (1975). The interaction of cyclic nucleotides and calcium in the control of cellular activity. Advances in Cyclic Nucleotide Research 6, Raven Press.
Birman H (2012). Bioactivities of plant flavonoids and the possible action mechanisms. Journal of Istanbul Faculty of Medicine 75:4649.

Bosgelmez Tinaz G, Ulusoy S, Ugur A, Ceylan O (2007). Inhibition of quorum sensing-regulated behaviors by Scorzonera sandrasica. Current Microbiology 55:114-118.

Briskin DP (2000). Medicinal plants and phytomedicines-linking plant biochemistry and physiology to human health. Journal of Plant Physiology 124:507-514.

Burda S, Oleszek W (2001). Antioxidant and antiradical activities of flavonoids. Journal of Agricultural and Food Chemistry 49:27742779.

Cai YZ, Sun M, Xing J, Luo Q, Corke H (2006). Structure-radical scavenging activity relationships of phenolic compounds from traditional Chinese medicinal plants. Life Science 78:2872-2888.

Campbell JD (2001). Life style, minerals and health. Medical hypotheses 57:521-531.

Canadanovic-Brunet JM, Cetkovic GS, Djilas SM, Tumbas VT, Savatovic SS, Mandic AI, Markov SL, Cvetkovic DD (2009). Radical scavenging and antimicrobial activity of horsetail (Equisetum arvense L.) extracts. International Journal of Food Science and Technology 44:269-278.

Deliorman-Orhan D, Özçelik B, Hoşbaş S, Vural M (2012). Assessment of antioxidant, antibacterial, antimycobacterial, and antifungal activities of some plants used as folk remedies in Turkey against dermatophytes and yeast-like fungi. Turkish Journal of Biology 36:672-686.

Domellöf M, Cohen RJ, Dewey KG, Hernell O, Rivera LL, Lönnerdal B (2001). Iron supplementation of breast-fed Honduran and Swedish infants from 4 to 9 months of age. Journal of Pediatrics 138:679-687.

Dorman DHJ, Bachmayer O, Kosar M, Hiltunen R (2004). Antioxidant properties of aqueous extracts from selected Lamiaceae species grown in Turkey. Journal of Agricultural and Food Chemistry 52:762-770.

Gallo FR, Multari G, Federici E, Palazzino G, Giambenedetti M, Petitto V, Poli F, Nicoletti M (2011). Chemical fingerprinting of Equisetum arvense L. using HPTLC densitometry and HPLC. Natural Product Research 25(13):1261-1270.

Gardiner P (1999). Chamomile (Matricaria recutita, Anthemis nobilis) Longwood Herbal Task Force. http://www.mcp.edu/herbal/ default. htm pp 21.

Gavamukulya Y, Abou-Elella F, Wamunyokoli F, Shemy H (2014). Phytochemical screening, anti-oxidant activity and in vitro anticancer potential of ethanolic and water leaves extracts of Annona muricata (Graviola). Asian Pacific Journal of Tropical Medicine 7:355-363.

Georgieff MK (2007). Nutrition and the developing brain: nutrient priorities and measurement. American Journal of Clinical Nutrition 85:614S-620S.

Guil-Guerrero JS, Gimenez-Gimenez A, Rodrguez-Garc I, Torija-Isasa ME (1998). Nutritional composition of Sonchus species (S. asper L., S. oleraceus L., and $S$. tenerrimus L.). Journal of the Science of Food 
and Agriculture 76:628-632.

Halici MG, John, Aksoy A (2005). Lichens of Erciyes Mountain (Kayseri, Turkey). Flora Mediterranea 15:567-580.

Hanachi P, SH G (2009). Using HPLC to determination the composition and antioxidant activity of Berberis vulgaris. European Journal of Scientific Research 29:47-54.

Hassan Z, Anwar Z, Khattak KU, Islam M, Khan RU, Zaman J, Khattak K (2012). Civic pollution and its effect on water quality of River Toi at District Kohat, NWFP. Research Journal of Environmental and Earth Sciences 4(3):334-339.

Heidari M, Sarani S (2012). Growth, biochemical components and ion content of chamomile (Matricaria chamomilla L.) under salinity stress and iron deficiency. Journal of the Saudi Society of Agricultural Sciences 11:37-42.

Henríquez C, Almonacid S, Chiffelle I, Valenzuela T, Araya M, Cabeza L, Simpson R, Speisky H (2010). Determination of antioxidant capacity, total phenolic content and mineral composition of different fruit tissue of five apple cultivars grown in Chile. Chilean Journal of Agricultural Research 70(4):523-536.

Ikinci N, Guner A (2007). Flora of the Gölcük Area (Bolu, Turkey). Turkish Journal of Botany 31:87-107.

Klevay LM (1975). Coronary heart disease: the zinc/copper hypothesis. American Journal of Clinical Nutrition 28:764-774.

Kocevar N, Odreman F, Vindign A, Grazio SF, Kome R (2012). Proteomic analysis of gastric cancer and immunoblot validation of potential biomarkers. World Journal of Gastroenterology 18:12161228.

Kon M, Ikeda T, Homma T, Akimoto T, Suzuki Y, Kawahara T (2010). Effects of acute hypoxia on metabolic and hormonal responses to resistance exercise. Medicine and Science in Sports and Exercise 42:1279-1785.

KukrıćZ, Topalić-TrivunovićL, Pavıčı́ć S,Žabıć M, MatošS, Davıdović A (2013). Total phenolic content, antioxidant and antimicrobial activity Of Equisetum arvense L. Chemical Industry and Chemical Engineering Quarterly 19:37-43.

Mebrahtu G, ZerabrukS (2011). Concentration and health implication of heavy metals in drinking water from urban areas of Tigray region, Northern Ethiopia. Momona Ethiopian Journal of Science 3(1):105-121.

Özcan M (2004). Mineral contents of some plants used as condiments in Turkey. Food Chemistry 84:437-440.

Parmer M (2012). Preliminary investigation of Herniaria incana Lam. determination of the total flavonoid content, antioxidant properties and free radical scavenging capacity. Master Thesis. Centre for Pharmacy University of Bergen (UiB) and Department of Chemistry. The Norwegian University of Science and Technology (NTNU) pp 73.

Passwater RA, Cranton EM (1983). Trace elements, hair analysis and nutrition. Keats Publishing Inc, New Canaan.

Raal A, Orav A, Gretchushnikova T (2014). Essential oil content and composition in Tanacetum vulgare $\mathrm{L}$. herbs growing wild in Estonia. Journal of Essential Oil Bearing Plants 17(4):670-675.
Rabiei Z, Rafieian-Kopaei M, Mokhtari S, Alibabaei Z, Shahrani M (2014). The effect of pretreatment with different doses of Lavandula officinalis ethanolic extract on memory, learning and nociception. Biomedicine and Aging Pathology 4:71-76.

Ruberto G, Barrata MT (2000). Antioxidant activity of selected essential oil components in two lipid model systems. Food Chemistry 69:167-174.

Sarac N, Ugur A (2007). Antimicrobial activities and usage in folkloric medicine of some Lamiaceae species growing in Mugla, Turkey. EurAsian Journal of BioSciences 4:28-37.

Schmidt BM (2012). Responsible use of medicinal plants for cosmetics. HortScience 47(8):985-991.

Shah A, Niaz A, Ullah N, Rehman A, Akhlaq M, Zakir M, Khan MS (2013). Comparative study of heavy metals in soil and selected medicinal plants. Journal of Chemistry 1-5.

Sharififar F, Pournourmohammadi S, Arabnejad M (2009). Immunomodulatory activity of aqueous extract of Heracleum persicum Desf. in mice. Iranian Journal of Pharmaceutical Research 8:287-292.

Sheded MG, Pulford ID, Hamed AI (2006). Presence of major and trace elements in seven medicinal plants growing in the SouthEastern Desert, Egypt. Journal of Arid Environments 66:210-217.

Shokrzadeh M, Saeedi Saravi SS (2010). The chemistry, pharmacology and clinical properties of Sambucus ebulus: A review. Journal of Medicinal Plants Research 4(2):95-103.

Singleton VL, Rossi JA (1965). Colorimetry of total phenolics with phosphomolybdic-phosphotungstic acid reagents. American Journal of Enology and Viticulture 16:144-158.

Skröder HM, Hamadani JD, Tofail F, Persson LA, Vahter ME, Kippler MJ (2015). Selenium status in pregnancy influences children's cognitive function at 1.5 years of age. Clinical Nutrition 34:923930.

Srivastava S, Srivastava M, Misra A, Pandey G, Rawat AKS (2015). A review on biological and chemical diversity in Berberis (Berberidaceae). Experimental and Clinical Sciences 14:247-267.

Stankovic MS, Niciforovic N, Mihailovic V, Topuzovic M, Solujic S (2012). Antioxidant activity, total phenolic content and flavonoid concentrations of different plant parts of Teucrium polium. subsp. polium. Acta Societatis Botanicorum Poloniae 81:117-122.

Trouillasa P, Callistea CA, Allaisc DP, Simonb A, Marfaka A, Delageb C, Durou JL (2003). Antioxidant, anti-inflammatory and antiproliferative properties of sixteen water plant extracts used in the Limousin countryside as herbal teas. Food Chemistry 80:399-407.

Tsuji PA, Canter JA, Rosso LE (2016). Trace minerals and trace elements. DOI: 10.1016/B978-0-12-384947-2.00699-1.

Tuberoso CI, Montoro P, Piacente S, Corona G, Deiana M, Dessì MA, Pizza C, Cabras P (2009). Flavonoid characterization and antioxidant activity of hydroalcoholic extracts from Achillea ligustica All. Journal of Pharmaceutical and Biomedical Analysis 50:440-448.

Turan M, Kordali S, Zengin H, Dursun A, Sezen Y (2003). Macro and micro mineral content of some wild edible leaves consumed in Eastern Anatolia. Acta Agriculturae Scandinavica 3:129-137. 
300

Veit M, Beckert C, Hohne C, Bauer K, Geiger H (1995). Probes for biological activity. New York: Academic Press, Interspecific and intraspecific variation of phenolics in the genus Equisetum subgenus Equisetum. Phytochemistry 38:881-891.

Wang SY, Zheng W (2001). Effect of plant growth temperature on antioxidant capacity in Strawberry. Journal of Agricultural and Food Chemistry 49:4977-4982.

Water Treatment Solutions (Cu) (1998). http://www.lenntech.com/ periodic/elements/.

Watts DL (1990). The nutritional relationships of calcium. Journal of Orthomolecular Medicine 5:612-666.

Woisky RG, Salatino A (1998). Analysis of propolis: some parameters and procedures for chemical quality control. Journal of Apicultural Research 37:99-105.

Wu Ch, Chen F, Wang X, Kim H, Guo-qing H, Haley-Zitlin V, Huang G (2006). Antioxidant constituents in fever few (Tanacetum parthenium) extract and their chromatographic quantification. Food Chemistry 96:220-227.
Yiğit D, Kandemir A (2002). Antimicrobial activity of some endemic plants (Salvia cryptantha, Origanum acutidens, Thymus sipyleus ssp. sipyleus). Journal of Education Faculty 4(2):77-81.

Yildirim E, Dursun A, Turan M (2001). Determination of the nutrition contents of the wild plants used as vegetables in upper Çoruh Valley. Turkish Journal of Botany 25:367-371.

Yücel E, Tapırdamaz A, Yücel Şengün İ, Yılmaz G, Ak A (2011). Determining the usage ways and nutrient contents of some wild plants around Kisecik Town (Karaman/Turkey). Biological Diversity and Conservation 4(3):71-82.

Zengin M, Ozcan MM, Cetin U, Gezgi S (2008). Mineral contents of some aromatic plants, their growth soils and infusions. Journal of the Science of Food and Agriculture 88:581-589. 